\title{
Editorial
}

\section{Stochastic Systems: Modeling, Optimization, and Applications}

\author{
Shuping He, ${ }^{1}$ Ju H. Park, ${ }^{2}$ Hao Shen, ${ }^{3}$ Zhengguang Wu, ${ }^{4}$ and Hongsheng Dai ${ }^{5}$ \\ ${ }^{1}$ School of Electrical Engineering and Automation, Anhui University, Hefei 230601, China \\ ${ }^{2}$ Department of Electrical Engineering, Yeungnam University, 214-1 Dae-Dong, Kyongsan 712-749, Republic of Korea \\ ${ }^{3}$ School of Electrical Engineering and Information, Anhui University of Technology, Ma'anshan 243002, China \\ ${ }^{4}$ National Laboratory of Industrial Control Technology, Institute of Cyber-Systems and Control, Zhejiang University, \\ Hangzhou 310058, China \\ ${ }^{5}$ Department of Mathematical Sciences, University of Essex, Wivenhoe Park, Colchester CO4 3SQ, UK
}

Correspondence should be addressed to Shuping He; shuping.he@ahu.edu.cn

Received 8 April 2014; Accepted 8 April 2014; Published 29 April 2014

Copyright (C) 2014 Shuping He et al. This is an open access article distributed under the Creative Commons Attribution License, which permits unrestricted use, distribution, and reproduction in any medium, provided the original work is properly cited.

Many stochastic systems, such as switching systems, Markovian jumping systems, jumping neural networks, T-S fuzzy jumping systems and network control systems, have arisen naturally in the mathematical modeling of phenomena spanning disciplines in the social sciences, natural sciences, and engineering. Much attention has been given to modeling, control optimization, robust filtering, signal processing, and real applications of such stochastic dynamical systems in the literature in recent years. This special issue provides a forum for researchers and practitioners to publish quality research work on modeling, optimization approaches, and their applications in the context of theory analysis and engineering developments. The accepted papers in this special issue include stochastic stability, stabilization and control optimization, stochastic optimization, particle swarm optimization, modeling and identification methods, signal processing, robust filtering, sliding mode control, multiagent systems, nonlinear systems, time-delayed systems, neural networks, T-S fuzzy systems, simulated annealing, and fault diagnosis methods.

In this special issue, we have accepted thirty-nine papers, of which six consider the stability and stabilization problems of stochastic systems. Twelve papers cover the problems of the controller design and relevant optimization algorithms. There are three articles that discussed the signal processing methods. One paper focuses on fault diagnosis and fault tolerant control for non-Gaussian singular time-delayed stochastic systems, and three consider the state estimation and filtering problems of discrete-time stochastic systems. Finally, fourteen papers study the stochastic systems modeling and identification methods.

Over the past few decades, the stochastic stability, stabilization, and control optimization problems have long been the mainstream of research topics. "Exponential synchronization of stochastic complex dynamical networks with impulsive perturbations and Markovian switching" by W. Zhou et al. studies the exponential synchronization for a class of Markovian switching networks with impulsive perturbations and "Almost sure asymptotical adaptive synchronization for neutral-type neural networks with stochastic perturbation and Markovian switching" by W. Zhou et al. concerns the almost sure asymptotical adaptive synchronization for a class of nonlinear Markovian switching networks. "Stabilization of discrete-time Markovian jump systems via controllers with partially mode-dependent characterization" by C. Zhai et al. developed a kind of stabilizing controller in terms of being partially mode-dependent for discrete-time Markovian jump systems. "Fuzzy stabilization for nonlinear discrete ship steering stochastic systems subject to state variance and passivity constraints" by W.-J. Chang et al. is concerned with a fuzzy stabilization methodology for nonlinear discretetime stochastic systems, subject to state variance constraint and passivity constraint. "Simple adaptive asymptotic tracking scheme for parametric strict-feedback nonlinear systems with 
additive disturbance" by Y. Wang et al. studies the adaptive asymptotic tracking scheme of a class of parametric strictfeedback nonlinear systems by using the backstepping technique. "Delay-dependent finite-time and $L_{2}$-gain analysis for switched systems with time-varying delay" by Q. Yang discusses the finite-time stability problem for switched systems. For the control optimization aspects, "Design of nonlinear robust rotor current controller for DFIG based on terminal sliding mode control and extended state observer" by G. Cai et al. considers a novel rotor current controller to enhance fault ride-through (FRT) capacities of grid-connected DFIG based on terminal sliding mode control scheme. "Dynamic intelligent feedback scheduling in networked control systems" by $\mathrm{H}$. Chen et al. studies a dynamic intelligent feedback scheduling strategy for networked control systems. By using the NN-GA approach, "Efficiency optimization for disassembly tools via using NN-GA approach" by G. Tian et al. addresses the disassembly efficiency optimization and the comparison for different disassembly tools. "Mean-varianceCvaR model of multiportfolio optimization via linear weighted sum method" by Y. Elahi and M. I. Abd Aziz proposes a new approach to optimizing portfolios to mean-variance-CVaR model. By both sides, bipolar-beamforming optimization, "Securing communication via transmission of artificial noise by both sides: bipolar-beamforming optimization" by Y. Zhou et al. considers the optimal design for the configuration to best utilize Alice and Bob's antenna and power resources. "Stochastic constriction cockroach swarm optimization for multidimensional space function problems" by I. C. Obagbuwa et al. examines the effect of stochastic constriction on cockroach swarm optimization algorithm performance, and "Analysis of population diversity of dynamic probabilistic particle swarm optimization algorithms" by Q. Ni and J. Deng measures the population diversity in PSO algorithms combined with some population diversity analysis methods. " $H_{\infty}$ enhanced control design of discrete-time Takagi-Sugeno state-multiplicative noisy systems" by D. Krokavec and A. Filasová and " $H_{\infty}$ control of singular Markovian jump systems with bounded transition probabilities" by H. Lin et al., respectively, study the $H_{\infty}$ control schemes for stochastic systems with unknown disturbances. "Intelligent integration between human simulated intelligence and expert control technology for the combustion process of gas heating furnace" by Y. C. Liu and Y. B. Liu explores a sort of strong robust control algorithm to improve the control quality of the combustion process of gas heating furnace. For the multiagent systems, "Leaderfollowing protocol design for switched multiagent systems with randomly occurring self-delay" by $M$. J. Park et al. designs a delay-dependent leader-following protocol for multiagent systems with both communication delay and randomly occurring self-delay, and "Fault tolerant consensus of multiagent systems with linear dynamics" by J. Li deals with the consensus problem of linear multiagent systems with actuator faults.

As is well know that the signal processing, estimation, and filtering problems are very useful in engineering applications. "Low-rank affinity based local-driven multilabel propagation" by $\mathrm{T}$. Li et al. presents a novel low-rank affinity based local-driven algorithm to robustly propagate the multilabels from training images to test images, and "Nonnegative signal decomposition with supervision" by $\mathrm{T}$. Li et al. gives a novel algorithm to numerically decompose mixed signals in a collaborative way. "SAR image despeckling with adaptive multiscale products based on directionlet transform" by $\mathrm{Y}$. Lu et al. proposes a new despeckling algorithm based on directionlets using multiscale products. For the estimation problems, "State estimation for nonlinear discrete-time systems with Markov jumps and nonhomogeneous transition probabilities" by S. Zhao et al. addresses the state estimation for a class of nonlinear discrete-time systems with Markov parameters and nonhomogeneous transition probabilities; "Multisensor estimation fusion of nonlinear cost functions in mixed continuous-discrete stochastic systems" by I. Y. Song et al. proposes the centralized and distributed fusion algorithms for estimation of nonlinear cost function in multisensory mixed continuous-discrete stochastic systems; "Suboptimal filtering of networked discrete-time systems with random observation losses" by S. Gao and P. Chen studies the remote filtering problem over a packet-dropping network. "Fault diagnosis and fault tolerant control for non-Gaussian singular time-delayed stochastic distribution systems" by L. Yao et al. proposes a new integrated fault diagnosis and fault tolerant control algorithm for non-Gaussian singular time-delayed stochastic distribution control system.

The problems of system modeling and identification have been extensively studied by many researchers, and many relevant results have been made for stochastic dynamical systems. "A multistep extending truncation method towards model construction of infinite-state markov chains" by K. Wang et al. proposes a multistep extending advanced truncation method towards model construction of CTMCs and implements it in the INFAMY model checker, and "A new GMRES $(m)$ method for Markov chains" by B.-Y. Pu et al. presents a class of new accelerated restarted GMRES method for calculating the stationary probability vector of an irreducible Markov chain. "Modeling of duck density and complex stocking time in rice-duck agroecosystems in terms of economic and ecological benefits" by D. Xiong et al. discusses the quantitative assessment and modeling of the duck density and complex stocking time in the rice-duck ecosystem, whereas "Noiseinduced transitions in a population growth model based on size-dependent carrying capacity" by N. Lumi et al. considers the stochastic dynamics of a population growth model with size-dependent carrying capacity. "Automated flare prediction using extreme learning machine" by Y. Bian et al. introduces a flare forecasting method which is the combination of the LR model and the ELM algorithm. "Punishment and feedback mechanism for the evolution game on small-world network based on varying topology" by Y. Shi et al. addresses the problem of the punishment and feedback mechanism for the evolution game on small-world network with varying topology. "Pricing decisions of two complementary products in a fuzzy environment" by L. Wang et al. analyzes the changes of the optimal retail pricing of two complementary products under two different decentralized decision scenarios, that is, Nash game case and Stackelberg game case. "Localization accuracy of range-only sensors with additive and multiplicative noise" by X. Shi et al. investigates the localization accuracy 
of range-only sensors with both additive and multiplicative noises, and "Integrating the supervised information into unsupervised learning" by P. Ling et al. presents an assembling unsupervised learning framework that adopts the information coming from the supervised learning process. "Comparison of multiple random walks strategies for searching networks" by Z. Zheng et al. investigates diverse random-walk strategies for searching networks, especially multiple random walks. "Molecular dynamics simulation of barnase: contribution of noncovalent intramolecular interaction to thermostability" by Z. Chen et al. focuses on the contribution of noncovalent intramolecular interaction to protein stability and how they affect the thermal stability of the enzyme, and "The simulation and minimization of loss probability in the tandem queueing with two heterogeneous channels" by M. Zobu et al. considers a stochastic model consisting of two heterogeneous channels and having no waiting room in front of each. For the application aspects, "Multiple model identification for a high purity distillation column process based on EM algorithm" by W. Xiong et al. introduces the multiple model based approach to model the high purity distillation column plant under the framework of the expectation maximization (EM) algorithm, and "Delay-aware online service scheduling in high-speed railway communication systems" by S. Xu et al. investigates the downlink service scheduling problem in relay-assisted highspeed railway communication systems.

It is necessary to point out that the selected topics and published papers are not a comprehensive representation of the area of this special issue. But the authors represent the rich and many faceted knowledge and they still hope the reader will find this special issue very useful.

\section{Acknowledgments}

We would like to express our great appreciation to all the authors of this special issue for their high quality contributions. All the reviewers' efforts in reviewing the papers are also very greatly acknowledged.

Shuping He
Ju H. Park
Hao Shen
Zhengguang Wu
Hongsheng Dai




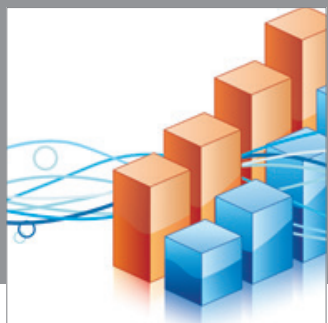

Advances in

Operations Research

mansans

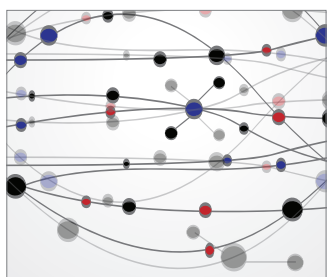

The Scientific World Journal
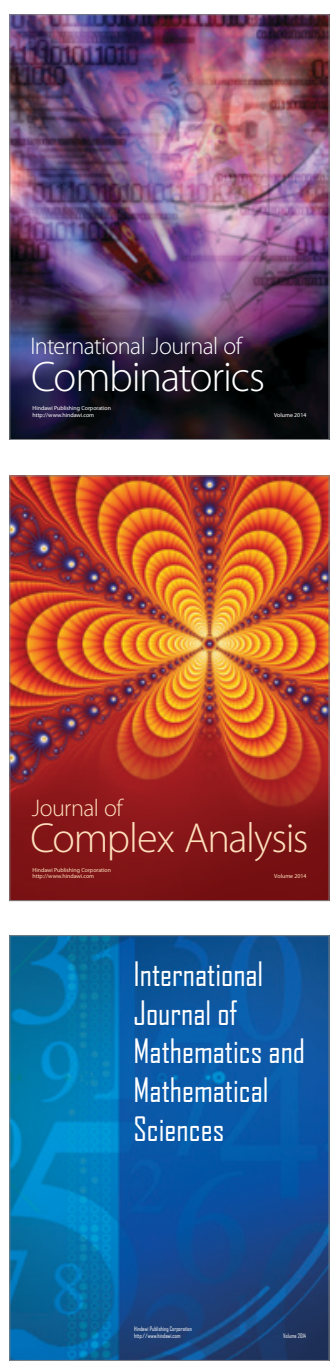
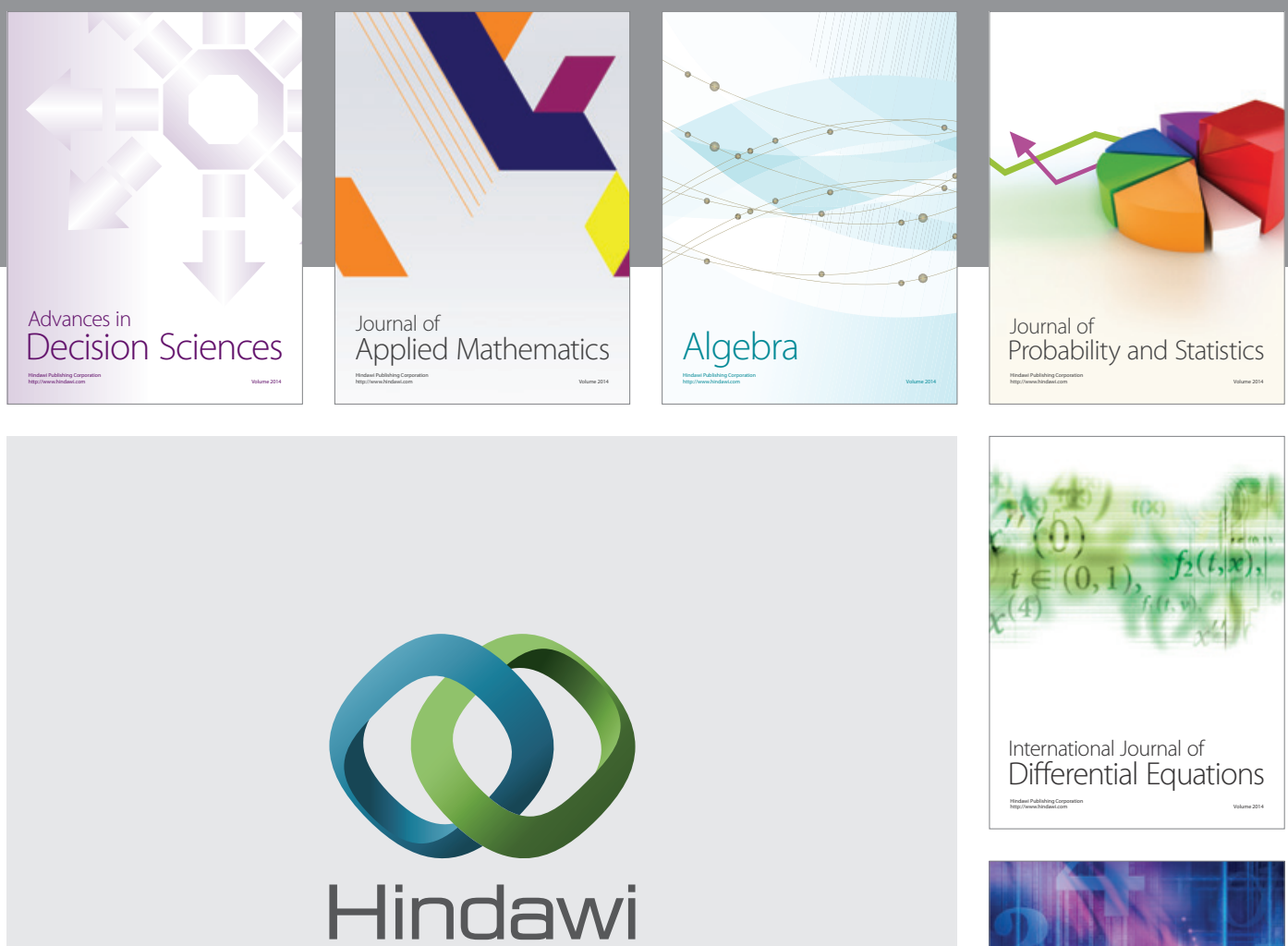

Submit your manuscripts at http://www.hindawi.com
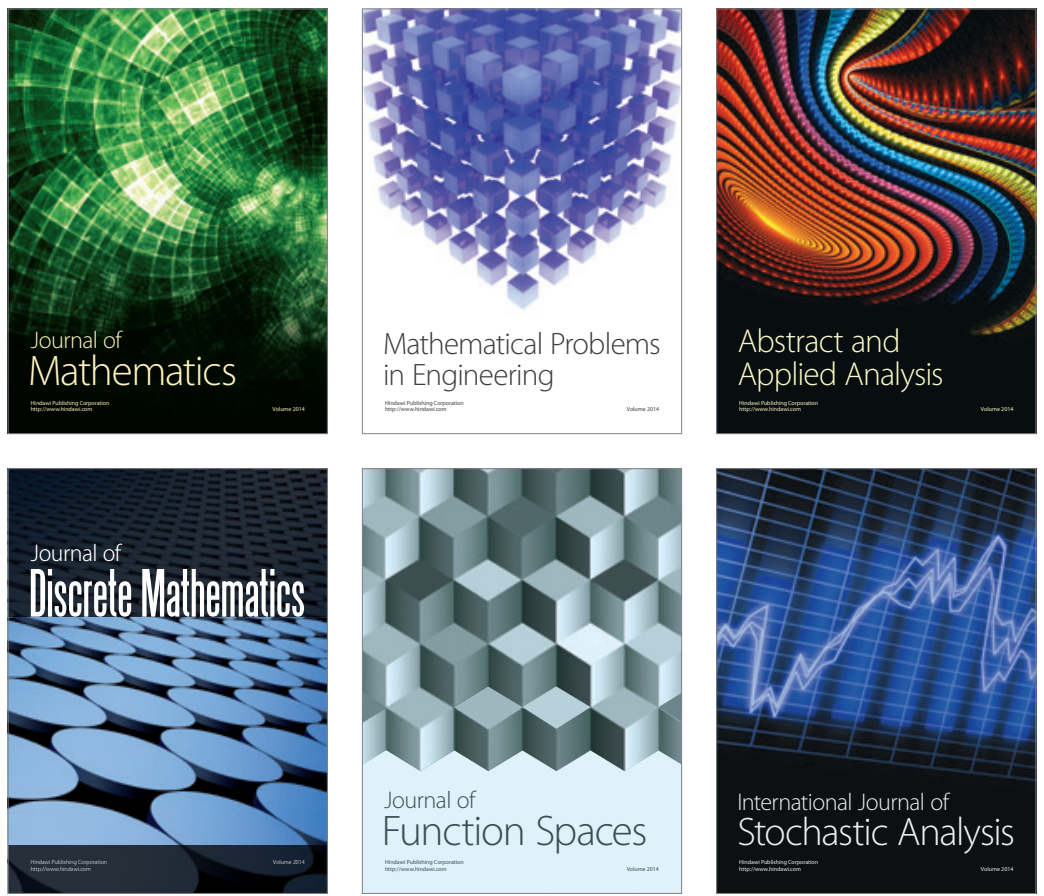

Journal of

Function Spaces

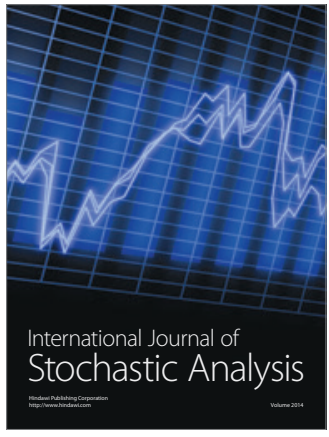

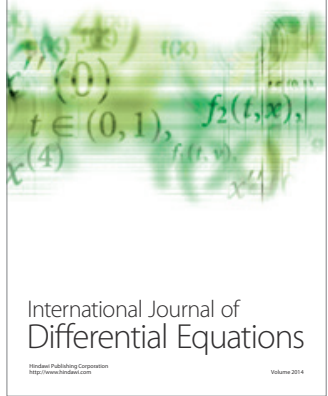
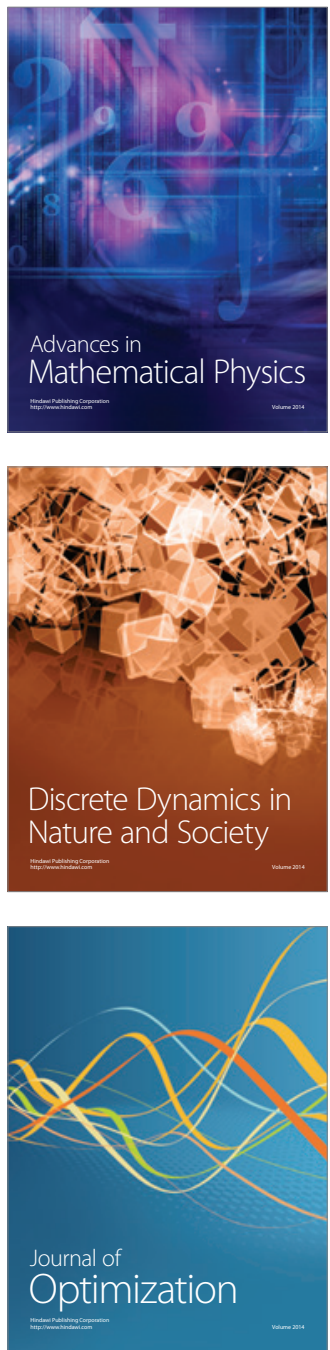